\title{
Os impactos das mudanças climáticas na Segurança Alimentar e Nutricional: uma revisão da literatura
}

\author{
The impacts of climate change on Food and Nutritional Security: \\ a literature review
}

Tais de Moura Ariza Alpino (https://orcid.org/0000-0001-5045-9483) ${ }^{1}$

Maíra Lopes Mazoto (https://orcid.org/0000-0001-5650-9402) ${ }^{1}$

Denise Cavalcante de Barros (http://orcid.org/0000-0001-5016-0844) ${ }^{2}$

Carlos Machado de Freitas (https://orcid.org/0000-0001-6626-9908) ${ }^{1}$

${ }^{1}$ Centro de Estudos e

Pesquisas em Emergências

e Desastres em Saúde, Fundação Oswaldo Cruz

(Fiocruz). Av. Brasil 4036, Sala 916, Manguinhos.

21041-361 Rio de Janeiro RJ Brasil.

arizatais@gmail.com

${ }^{2}$ Escola Nacional de Saúde Pública Sérgio Arouca,

Fiocruz. Rio de Janeiro RJ

Brasil.

\begin{abstract}
The interface between Climate Changes and Food and Nutrition Security (FNS) has been standing out in the sustainable development agenda since the early 1990's. Since then, studies show that climate changes have negative effects on the FNS, aggravated by poverty and social inequality. The purpose of this paper is to perform a review evidencing the relationships between climate changes and FNS. The research was carried out in PubMed using the descriptors "climate change and food security" on the headline, selecting only papers in Portuguese, Spanish, and English languages, and with a direct relation to the themes. The main impacts of climate changes on the FNS were related to the access, production, nutritional quality, and volatility of food prices. The studies also indicated mitigation/adaptation strategies to the effects of climate changes on the FNS, as well as a geographic panorama of the publications with fields of study in Africa and Asia, continents marked by social inequality and poverty. Climate changes affect the dimensions of FNS, especially in poorer populations in situation of social inequality. The relevance of the themes raises concern on the urgency of higher investments in public policies, studies, and research on the subject around the world.

Key words Climate changes, Food and Nutrition Security, Poverty, Inequality
\end{abstract}

Resumo A interface entre as Mudanças Climáticas e a Segurança Alimentar e Nutricional (SAN) tem se destacado na agenda de desenvolvimento sustentável desde o início da década de 1990. Desde então, estudos demonstram que as mudanças climáticas possuem efeitos negativos na SAN, potencializados pela pobreza e desigualdade social. O objetivo deste artigo é realizar uma revisão relacionando mudanças climáticas e SAN. A pesquisa foi realizada no PubMed utilizando os descritores "climate change and food security" no título, selecionando somente artigos em português, espanhol e inglês e com relação direta com os temas. Os principais impactos das mudanças climáticas na SAN foram no acesso, produção, qualidade nutricional e volatilidade dos preços dos alimentos. Estratégias de mitigação/adaptação aos efeitos das mudanças climáticas na SAN também foram apontadas nos estudos, além de um panorama geográfico das publicações com domínio de estudos na África e Âsia, continentes marcados por desigualdade social e pobreza. As mudanças climáticas afetam as dimensões da SAN, especialmente em populações mais pobres e em situação de desigualdade social. A relevância dos temas suscita a premência de maior investimento em políticas públicas, estudos e pesquisas acerca da temática no mundo.

Palavras-chave Mudanças climáticas, Segurança Alimentar e Nutricional, Pobreza, Desigualdade 


\section{Introdução}

Os primeiros estudos sobre os impactos das Mudanças Climáticas na Segurança Alimentar e Nutricional (SAN) surgiram na década de 1990 e podem ser divididos em: fase neutra (19942005): em que as mudanças climáticas não causariam efeitos negativos na SAN. Acreditava-se que as mudanças climáticas criariam ganhadores e perdedores, mas, ao final, a produção de alimentos "daria conta do recado"; e uma fase negativa (2005 até hoje): aumenta a especulação de que as mudanças climáticas e seus efeitos estão contribuindo para a fome no mundo ${ }^{1}$.

O clima no planeta está claramente mudando caracterizado por eventos como: ondas de calor, inundações, secas, aumento do nível dos mares e poluição atmosférica, que afetam a saúde direta ou indiretamente, causando impactos diversos como aumento de doenças respiratórias e transmissíveis, inúmeros danos, doenças e agravos à saúde provocados por desastres, além de óbitos, comprometendo no médio e/ou longo prazo a SAN.

A SAN é definida como a capacidade de garantir a todos acesso a alimentos básicos de qualidade e em quantidade suficiente, sem comprometer as outras necessidades essenciais. Possui quatro dimensões: acesso, disponibilidade, utilização e estabilidade ${ }^{2,3}$.

Diante deste contexto, estudos afirmam que as mudanças climáticas possuem efeitos negativos na SAN. Estes são influenciados por múltiplos determinantes: pobreza, nível de instrução, desemprego, aumento do preço dos alimentos, acesso deficiente aos alimentos, falha nos direitos à propriedade/terra e trabalho, além do clima/ ambiente $^{4-8}$ e dependerão da capacidade de responder e/ou se adaptar ao novo cenário.

O número de eventos extremos, incluindo calor extremo, secas, inundações e tempestades, dobrou desde o início dos anos 1990, com uma média de 213 ocorrências/ano no período de 1990 a $2016^{3}$. Alguns riscos foram identificados: aumento da variabilidade das precipitações (inundações e secas mais frequentes e extremas) e no escoamento de rios; redução na produtividade das plantações; aumento da temperatura global $\left(1,8^{\circ} \mathrm{C}\right.$ para $\left.4^{\circ} \mathrm{C}\right)$; na composição do solo ${ }^{5,9,10}$ e alterações nos ciclos de vetores de doenças afetando o estado de saúde da população $0^{3,11-13}$. Diante deste novo cenário, alguns cultivos mostram-se mais sensíveis do que outros: o trigo é mais sensível a mudanças de temperatura do que o arroz ${ }^{14}$, por exemplo.
Os efeitos negativos das mudanças climáticas na SAN têm sido especialmente estudados em países em desenvolvimento com evidências da sua contribuição para o agravamento das diferentes formas de má nutrição: desnutrição, deficiências nutricionais e sobrepeso/obesidade. Nos países desenvolvidos o foco é a qualidade e segurança dos alimentos ${ }^{3,15}$.

As mudanças climáticas podem causar riscos a SAN por meio de reduções na disponibilidade de alimentos, acesso, utilização e estabilidade do sistema alimentar, o que, combinado com a alta demanda, eleva os preços dos alimentos. O sistema alimentar instável, com baixa oferta de alimentos in natura e preços elevados, aumenta a busca por alimentos ultraprocessados e processados, que traz à tona uma outra vertente da insegurança alimentar e nutricional: o sobrepeso/obesidade ${ }^{16}$. Ou seja, as mudanças climáticas possuem interface com a má nutrição e com a insegurança alimentar e nutricional: desnutrição/ déficit nutricional e sobrepeso/obesidade, reforçando a necessidade de políticas públicas intersetoriais que compreendam os determinantes que influenciam as escolhas alimentares da população e suas consequências má nutrição, dando respostas para além do setor saúde ${ }^{17}$.

No ano de 2017 registrou-se um aumento de $25 \%$ da fome aguda e crônica no mundo em relação aos 2 anos anteriores devido, principalmente, a fatores relacionados ao clima (aumento das secas, por exemplo) ${ }^{3}$.

O conhecimento sobre os impactos das mudanças climáticas na agricultura tem expandido significativamente nos últimos 20 anos. No entanto, observa-se uma carência de estudos sobre a interface das mudanças climáticas com a SAN, especialmente em países em desenvolvimento, apontados pela literatura como os mais vulneráveis a tais eventos. Diante deste contexto, o presente artigo tem como objetivo realizar uma revisão da literatura científica, traçando um panorama da relação entre as mudanças climáticas e a SAN, visando contribuir para o alcance dos objetivos do desenvolvimento sustentável $(\mathrm{ODS})^{18}$.

\section{Metodologia}

A pesquisa foi realizada na base de dados PubMed (http://www.ncbi.nlm.nih.gov/pubmed) no período de agosto a outubro de 2018, utilizando combinação dos descritores: [Climate change and food security] no título. 
Considerando que este artigo de revisão tem como objetivo fazer uma primeira aproximação do tema das mudanças climáticas e da SAN nas Ciências da Saúde optamos por utilizar somente o PubMed por este ser a principal base de periódicos e livros do mundo no campo da Saúde Pública/Coletiva.

Os critérios de inclusão foram: (1) somente artigos originais; (2) idiomas português, espanhol e inglês; (3) ter relação direta com os temas estabelecidos. Excluiu-se artigos de revisão, resumos de congressos, monografias, dissertações, teses, capítulos de livro.

Para elaboração da revisão avaliou-se inicialmente os títulos, seguido da leitura dos resumos e, posteriormente, a leitura na íntegra dos estudos. Identificou-se, inicialmente, 38 artigos originais e após leitura dos resumos, excluiu-se 1. Trinta e sete artigos foram lidos na íntegra e 3 foram excluídos por não apresentarem relação direta com os temas definidos. No total 34 artigos foram considerados elegíveis para esta revisão (Figura 1).

\section{Resultados e discussão}

Os resultados encontrados foram organizados em 5 (cinco) categorias analíticas: acesso, disponibilidade, utilização, estabilidade e estratégias de adaptação/mitigação. Em seguida, foi organizado um panorama geográfico dos estudos.

\section{Acesso}

Acesso é uma das dimensões da SAN e aborda a presença de recursos econômicos e físicos, para adquirir os alimentos disponíveis para atender suas necessidades ${ }^{2}$. Quinze publicações s, $^{4,11,12,15,19-28}$ discutiram como as mudanças climáticas podem causar impactos diretos nas plantações e consequente redução na produção de alimentos. Isso pode afetar $\mathrm{o}$ acesso aos alimentos, principalmente pela falta de renda consequente do desemprego dos agricultores que perdem seus animais (pecuária ${ }^{27}$, suas terras e/ou com a redução da produtividade das mesmas ${ }^{12,15,19,20,24-26}$, pelo aumento dos preços dos alimentos básicos decorrente da queda da produtividade e aumento da demanda ${ }^{12,15,20,26}$, e, a dificuldade na distribuição e armazenamento da produção ${ }^{22,23}$.

Fischer et al. ${ }^{29}$ apontam que os preços dos alimentos e as temperaturas irão aumentar concomitantemente e de forma moderada até 2050, afetando negativamente a quantidade, a qualidade e a diversidade dos alimentos consumidos no mundo ${ }^{3}$.

De maneira mais ampla Schmidhuber e Tubiello ${ }^{5}$ discutem conceito de acesso relacionado com a garantia de direitos políticos, econômicos, sociais de modo que as populações possam adquirir uma alimentação adequada em qualidade e quantidade nutricional. Assim, unindo fatores e direitos econômicos e sociais ao acesso aos alimentos.

Um estudo de caso realizado em Madagascar (África) aponta que os altos níveis de pobreza, infraestrutura precária e falta de apoio financeiro e técnico de agricultores são fatores que os tornam potencialmente mais vulneráveis a riscos climá$\operatorname{ticos}^{24}$. Na África do Sul comunidades rurais dependentes da agricultura enfrentam também um risco instantâneo de quebra de safra e perda de gado, e, consequentemente, a fome e a desnutrição aumentam porque a maioria dessas pessoas obtém renda de produtos agrícolas ${ }^{30}$.

Sendo assim, o direito à alimentação por meio do acesso não é garantido, especialmente

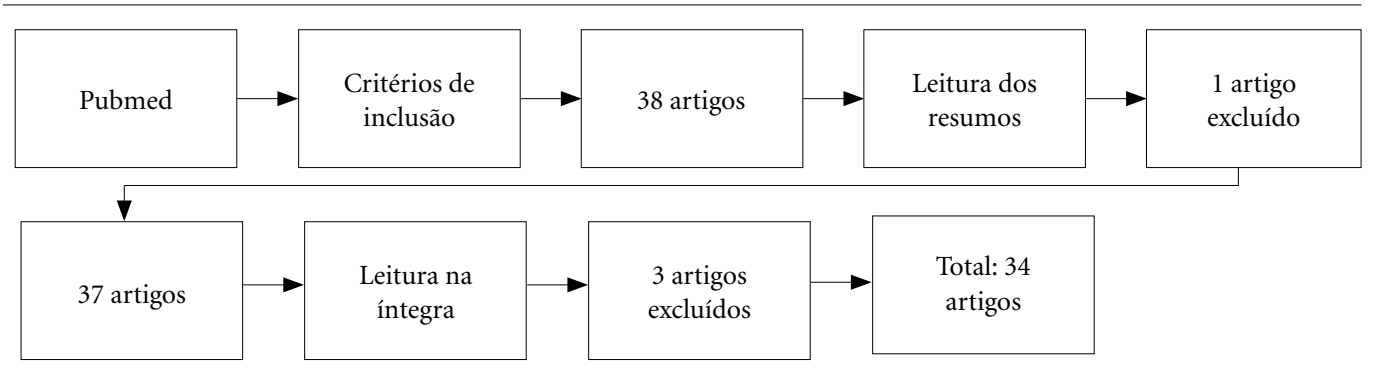

Figura 1. Esquema representativo da busca de artigos para o estudo. 
em países com vulnerabilidades socioambientais (países de baixa e média renda que sofrem com maiores efeitos das mudanças climáticas), resultando em múltiplas formas de má nutrição (deficiência de micronutrientes, desnutrição e obesidade) na população ${ }^{2,3}$.

A desnutrição como resultado da falta de acesso à alimentação é um efeito esperado, porém a obesidade traz para discussão um novo padrão de alimentação, com maior consumo de alimentos ultraprocessados, alimentos com baixo valor nutricional, afetando diretamente a SAN ${ }^{15}$.

\section{Disponibilidade}

A categoria de disponibilidade foi a que apresentou maior número de publicações: 26 $\operatorname{artigos}^{1,5,11,12,15,19,20-25,28,31-43}$, sendo que a maioria destes aborda, especialmente, os efeitos diretos e indiretos das mudanças climáticas na produção de alimentos $5,12,15,19,20,22-25,31,33,38,39$, que variam de região para região, considerando a vulnerabilidade do território e população. Os efeitos diretos são pelas alterações nas condições agroecológicas (aumento da temperatura, da frequência e severidade de eventos extremos, do nível dos rios, das concentrações de $\mathrm{CO}_{2}$ na atmosfera, erosão nos solos, mudança do ciclo das águas, alterações do ciclo de vetores e pragas) $)^{34,40,43}$ que reduzem a produtividade das plantações ${ }^{11,25,37}$ e perda de animais $^{11,12,19,32}$. Os indiretos afetam a distribuição de renda dos produtores. Discute-se também a relação dos efeitos das mudanças climáticas com o crescimento da população (maior demanda) e menor disponibilidade de alimentos ${ }^{20,35}$.

A dimensão de disponibilidade corresponde à existência de quantidades suficientes de alimentos de qualidade adequada, fornecidos através da produção do país ou de importações, incluindo ajuda alimentar ${ }^{2}$.

O crescimento populacional e as mudanças nos hábitos alimentares, com destaque para o maior consumo de carne, produtos lácteos e ultraprocessados, aumentaram a demanda por alimentos. No entanto, o aumento de fatores não alimentares, como utilização de biocombustíveis, urbanização, erosão do solo e mudanças climáticas influenciam em potencial a SAN, especialmente a disponibilidade de alimentos ${ }^{22}$.

Discute-se ainda os efeitos diretos e indiretos das mudanças climáticas na produtividade agrícola: diretos - variabilidades climáticas (secas e inundações); e, indireta - através de pragas e doenças, aumento médio no nível do mar e mudanças na disponibilidade de água ${ }^{44}$.
Há fortes evidências de que as mudanças climáticas são impulsionadas pelo El Niño e desempenham um papel fundamental na diminuição dos rendimentos das plantações, especialmente de arroz, trigo e milho ${ }^{1,3,12,15,45,46}$. Além disso podem diminuir a área e qualidade das terras cultiváveis, principalmente na África ${ }^{5}$, e afetar a produção de alimentos pesqueiros e a pecuária ${ }^{12}$.

A discussão em torno das vulnerabilidades é relevante quando aborda-se a interface entre as mudanças climáticas e a SAN. Países como a África do Sul, que apresentam uma sobreposição de vulnerabilidades (propensão à seca, pobreza, desigualdades sociais, falta de acesso à educação, ausência de políticas públicas, etc.), sofrerão os efeitos das variabilidades climáticas na SAN de forma mais frequente e intensa ${ }^{20}$. Estudos afirmam que toda a África e Sul da Ásia podem sofrer uma redução de $8 \%$ na produção de alimentos até $2050^{25}$.

Uma recente publicação da Lancet $^{47}$ apontou duas formas de má nutrição, desnutrição e obesidade, e, as mudanças climáticas como três pandemias simultâneas que resultam em efeitos na saúde humana. Essas três pandemias representam a Sindemia Global, que afeta a maioria das pessoas em todos os países e regiões do mundo. Por exemplo, os sistemas alimentares não apenas impulsionam as pandemias de obesidade e desnutrição, mas também geram de 25-30\% das emissões de gases do efeito estufa, desmatamento, perda de biodiversidade e degradação do solo, fatores estes que influenciam nas mudanças climáticas. Por sua vez, as mudanças climáticas afetam os sistemas alimentares resultando em alterações no consumo e hábitos alimentares e consequentemente na má nutrição e na SAN.

Considerando este contexto, o aumento da desnutrição é maior nos países de baixa e média renda devido à redução na produção de alimentos causada por eventos extremos climáticos. Os países mais ricos, por conta dos processos de urbanização, industrialização acelerada e consequentemente maior emissão de gases de efeito estufa, apresentam maior prevalência de obesidade em comparação com países de baixa e média renda. As mudanças nos hábitos alimentares também devem ser consideradas, como o aumento no consumo de laticínios, carnes e ultraprocessados que, por sua vez, também favorecem a emissão de gases de estufa.

Embora os efeitos diretos das mudanças climáticas na produtividade das culturas sejam amplamente estudados, ainda existem limitações importantes no estudo dos impactos na disponi- 
bilidade de alimentos. Primeiro, estes estudos que mapeiam os efeitos negativos das mudanças climáticas nas plantações possuem foco nos principais cereais e algumas raízes e tubérculos. Segundo, os estudos não avaliam os efeitos das mudanças climáticas na pecuária (mudanças na produtividade da pastagem, na qualidade do pasto e na alimentação do gado). Por último, os estudos de plantações são menos precisos para extremos climáticos, que podem ter consequências ainda mais importantes para os rendimentos das culturas ${ }^{25}$.

\section{Utilização}

A utilização foi abordada em 9 arti$\operatorname{gos}^{12,15,20,22,25,27,28,38,48}$, focando no efeito das mudanças climáticas sobre o consumo de alimentos, qualidade nutricional e seu valor social. A qualidade nutricional ${ }^{12,15,22,38,48}$ pode ser afetada tanto pela redução nas concentrações de micronutrientes (zinco, ferro, fósforo, potássio, cálcio, enxofre, magnésio, cobre e manganês) em diferentes culturas alimentares, como pela redução da ingestão de frutas, hortaliças, nozes, sementes e peixes, alimentos ricos em nutrientes essenciais para o organismo $0^{7,12,38,49}$.

Embora os impactos na utilização dos alimentos sejam menos estudados, comparado as demais dimensões, estudos sugerem que as mudanças climáticas afetam negativamente a qualidade nutricional dos alimentos ${ }^{3}$, o que influencia as condições de vida, o estado nutricional e a saúde dos indivíduos ${ }^{2}$.

Myers et al. ${ }^{12}$ sugerem que as mudanças climáticas causam uma redução na produção/colheita de alimentos, incluindo o setor pesqueiro, o que torna milhões de pessoas mais vulneráveis a déficits nutricionais, principalmente os mais pobres, afetando diretamente sua saúde.

Assim como as dimensões anteriores, a utilização contribui para estados de má nutrição (desnutrição, deficiências de micronutrientes e obesidade) e, consequentemente, para alterações no crescimento, desenvolvimento cognitivo, sistema imunológico e maior risco de doenças não transmissíveis (hipertensão arterial, diabetes mellitus, câncer), especialmente em grupos vulneráveis como recém-nascidos, crianças menores de 5 anos, lactentes e idosos ${ }^{2}$. Crianças em situação de pobreza tendem a ter menor estatura e consequências nutricionais severas como resultado das mudanças climáticas no acesso, produção e utilização dos alimentos ${ }^{27}$.

Estudos realizados em Bangladesh e na Indonésia mostraram que as mudanças climáticas afetaram a produção e causaram aumento no preço do arroz, situação associada ao aumento de prevalência de baixo peso em crianças, afetando diretamente o estado nutricional ${ }^{2,3}$.

\section{Estabilidade}

Estabilidade corresponde à garantia de disponibilidade, acesso e uso adequado de alimentos por indivíduos e, portanto, engloba as outras 3 dimensões. Para esta análise optou-se por selecionar os artigos que focaram na volatilidade dos preços dos alimentos.

Esta foi a categoria com menor número de publicações: 6 artigos $^{4,5,12,25,28,50}$, que discutiram a relação das mudanças climáticas com a redução da produção agrícola e a quantidade de alimentos disponíveis nos sistemas alimentares o que altera os preços dos alimentos (volatilidade) $)^{5,12,25}$, especialmente em áreas rurais ${ }^{50}$.

De maneira geral, os processos de urbanização, o esgotamento dos recursos naturais, ocupação do solo, desmatamentos, mudanças climáticas e desastres naturais são fatores que afetam o acesso, a disponibilidade e utilização dos alimentos, e, por conseguinte, a estabilidade dos sistemas alimentares ${ }^{2}$.

As mudanças climáticas afetam a estabilidade de sistemas alimentares pois o clima é um determinante importante nos padrões de produção, oferta e demanda de alimentos, resultando nas variabilidades a curto e longo prazo dos preços, principalmente em populações mais pobres e vulneráveis ${ }^{2,25}$. Com a ocorrência dos desastres naturais, como as secas e inundações, a produção de alimentos é prejudicada e/ou ainda há uma redução nas áreas cultiváveis, trazendo impactos relevantes para os sistemas alimentares. Cerca de $25 \%$ dos impactos econômicos negativos dos desastres recaem sobre o setor agrícola, o que se torna uma ameaça à SAN.

A variabilidade dos preços dos alimentos impacta diretamente as populações mais vulneráveis visto que o gasto com alimentos representa uma maior proporção de sua renda e estes tendem a ter menor capacidade/possibilidade de mudar seus padrões de consumo, forçando-os a consumir alimentos com menor qualidade nutricional. Por outro lado, a queda no preço dos alimentos faz com que pequenos produtores sofram com flutuações significativas em sua renda e tenham menor acesso a tecnologias, infraestrutura e recursos para lidar com a volatilidade dos preços, gerando custos significativos para os sistemas alimentares ${ }^{2}$. 
A elasticidade do preço dos alimentos associada a falta de estabilidade dos sistemas alimentares como resultados das mudanças climáticas resulta na redução do consumo de todos os grupos de alimentos e, consequentemente, em situações de insegurança alimentar e nutricional, especialmente em populações mais vulneráveis.

Dessa forma, pode-se afirmar que as mudanças climáticas também influenciam na estabilidade do sistema alimentar visto que esta dimensão é transversal às outras três (acesso, disponibilidade, utilização). Esta situação gera maior consumo de alimentos com pouca qualidade nutricional, como os alimentos processados e ultraprocessados, afetando diretamente o estado nutricional dos indivíduos (maior prevalência de sobrepeso/ obesidade). Além disso, as alterações na estabilidade causam menor acesso e produção de alimentos (quantidade) ocasionando deficiências nutricionais e desnutrição ${ }^{16,47}$. Ambas as situações alteram a SAN resultando nas diferentes formas de má nutrição.

Dados recentes demonstram que 672 milhões de indivíduos adultos estão obesos $(13,3 \%$ do total da população desta faixa etária) e padecem das consequências do excesso de peso. Ao mesmo tempo, 821 milhões de pessoas em todo o mundo estão famintas, enquanto 2 bilhões vivem em situação de severa/moderada insegurança alimen$\operatorname{tar}^{47,51}$.

Vale destacar que as mudanças climáticas podem aumentar o número de pessoas com risco de fome em $20 \%$ até $2050^{52}$, o que acarretará o não cumprimento do $2^{\circ}$ ODS: "Acabar com a fome, alcançar a segurança alimentar e melhoria da nutrição e promover a agricultura sustentável"18.

A Figura 2 traz os principais impactos das mudanças climáticas na SAN.

\section{Estratégias de adaptação/mitigação}

A categoria estratégias de adaptação/mitigação foi a segunda com maior número de publicações, 24 artigos $^{4,5,15,20-25,28,31,34-39,41,43,49,50,53-55}$.

As estratégias de adaptação/mitigação visam tornar os sistemas alimentares mais resilientes às mudanças climáticas no futuro, a saber: $\mathrm{a}$ implementação de políticas públicas ${ }^{39}$, planos de desenvolvimento agrícola, leis de proteção do mercado, abertura de mercados locais e subsídios agrícolas $^{5,41}$, bem como a manutenção e recuperação dos solos afetados ${ }^{5,20,35,39}$; adaptação do plantio aos períodos de precipitação; aumento do uso de irrigação $0^{20}$; aumento da variedade e rotação de culturas ${ }^{37}$; utilização de fertilizantes inorgânicos e da agricultura de baixo carbono; fortificação e biofortificação; manejo de pragas; reflorestamento; inovações tecnológicas (genética, biotecnologia e agronomia) e uso de biocombustíveis ${ }^{5,15,22,24,25,28,31,36,43,49,50,53,55}$.

A discussão sobre a união das necessidades de adaptação relacionadas à manutenção da produtividade agrícola e da integridade do ecossistema, visando a redução da pobreza, proteção da produção agrícola e dos serviços ecossistêmicos, também se fez presente nos estudos como uma estratégia de adaptação e mitigação (agrossilvicultura $)^{54}$.

Os artigos citam também a necessidade de implementação de sistemas de alerta e alarme e ferramentas de monitoramento eficazes para auxiliar nas tomadas de decisão para proteção da produção agrícola ${ }^{28}$. Somado a isso, aponta-se como necessária a elaboração de planos integrados para prevenção, proteção, resposta e reabilitação das áreas expostas com participação social $^{36,39,50}$. No entanto, isso requer a sensibilização e capacitação da sociedade civil acerca dos riscos e ações de preparação, resposta e comunicação de risco por meio de práticas educativas e sustentáveis $^{21,23,24,38}$.

Lipper et al..$^{56}$ apontam a importância de ações coordenadas envolvendo múltiplos atores (agricultores, sociedade civil, pesquisadores, setor privado e gestores) para a construção da resiliência às mudanças climáticas e propõem a implementação da "climate-smart agriculture" (CSA). A CSA é uma agricultura resiliente/resistente ao clima ancorada em práticas agrícolas sustentáveis que visam o aumento da produtividade e da renda, da resiliência dos meios de subsistência e dos ecossistemas e a redução/remoção de gases dos efeitos estufa da atmosfera ${ }^{57}$, contribuindo de forma transversal com os ODS. Segundo a FAO, a CSA é a chave para a SAN frente aos desafios das mudanças climáticas ${ }^{58}$.

A transformação do setor agrícola é urgente e fundamental para a resposta às mudanças climáticas. Segundo a $\mathrm{FAO}^{59}$, para isso é necessário o aumento dos investimentos em pesquisas sobre os efeitos das mudanças climáticas na agricultura; no monitoramento de dados/informações climáticas e sistemas de alerta/alarme que permitam a antecipação de ações e redução dos danos (mapeamento, modelos estatísticos, etc.); além de crédito agrícola, disseminação de conhecimento sobre o assunto, envolvimento do setor privado para a implementação de técnicas agrícolas sustentáveis, acesso a seguros agrícolas e organização de cooperativas ${ }^{58,59}$. 


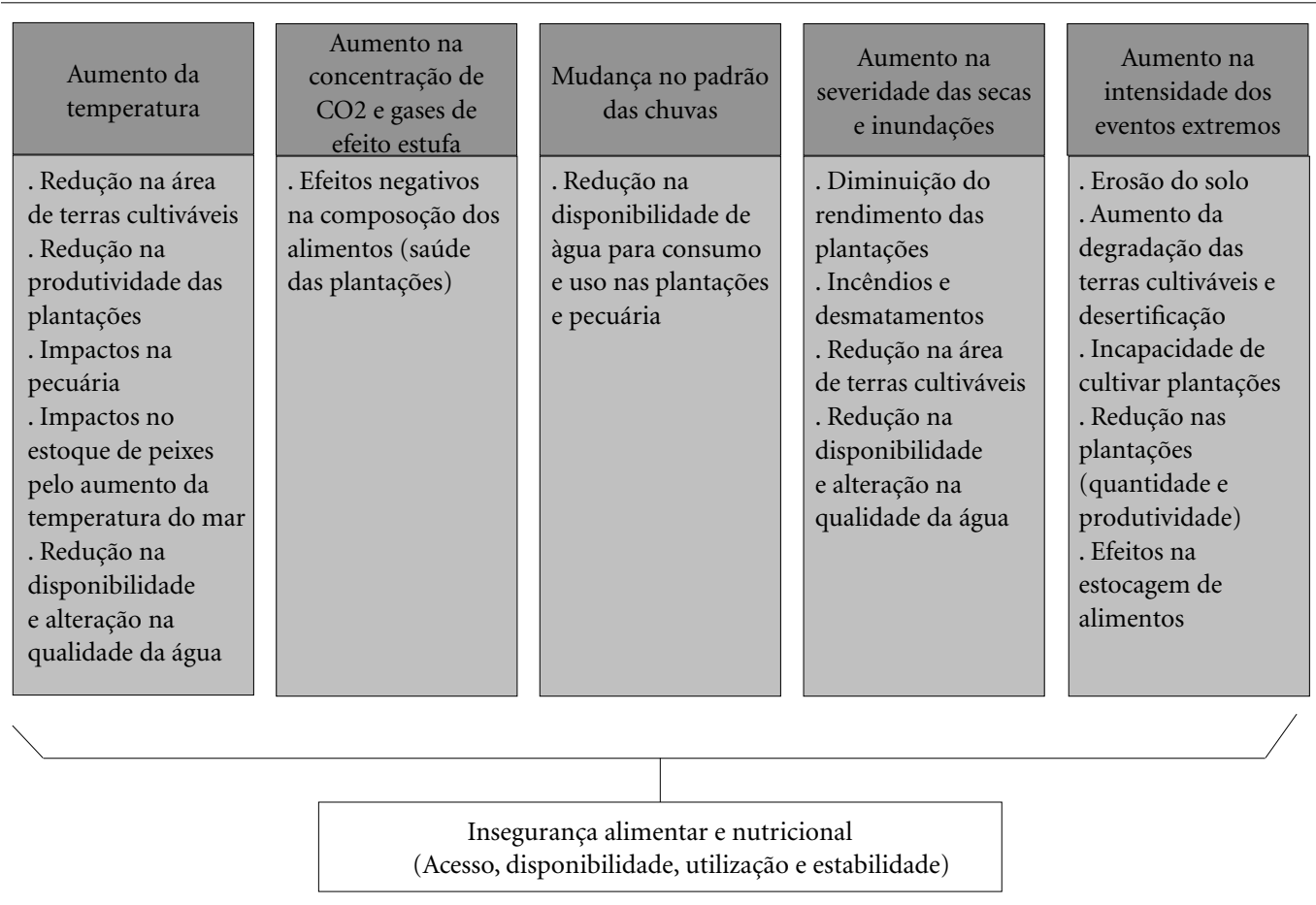

Figura 2. Principais impactos das mudanças climáticas na SAN.

Fonte: Adaptada de Raiten e Aimone ${ }^{16}$ e Masipa $^{20}$.

É importante destacar que "o combate às mudanças climáticas" é um dos 17 ODS das agendas governamentais até $2030^{18}$.

Considerando as categorias analíticas discutidas acima, elaborou-se um quadro contendo uma síntese dos principais resultados encontrados no presente estudo (Quadro 1).

\section{Locais de estudo}

Visando contextualizar o tema no cenário internacional, analisou-se também os locais de publicação dos 34 artigos considerados elegíveis para o estudo (Figura 3 ).

A Figura 3 mostra que os estudos encontrados foram realizados predominantemente em países da África e Ásia, os 2 (dois) continentes mais populosos do mundo, sendo que alguns artigos elegíveis para o estudo (14 no total) não continham informação sobre localização.

\section{África}

O continente africano é o segundo continente mais populoso do mundo, com mais de $60 \%$ de sua população vivendo na área rural. É pobre, pouco industrializado e possui baixos índices de desenvolvimento econômico. Suas riquezas representam $1 \%$ do Produto Interno Bruto (PIB) mundial e o PIB per capita gira em torno de U\$ 915,00 . Os principais problemas são: fome, epidemias (HIV, principalmente) e conflitos étnicos. Os indicadores sociais também não são bons, com uma população de aproximadamente $40 \%$ de analfabetos e uma taxa de mortalidade infantil que chega a 100-200 mil óbitos por cada 1000 nascimentos (a mais alta do mundo). Somado a isso, o continente abriga $1 / 3$ da população mundial em condições de extrema pobreza e 7 dos 10 países com maior desigualdade no mundo estão localizados no continente africano ${ }^{60}$.

Com relação a SAN, o Mapa da Fome publicado pela World Food Programme (WFP) em $2018^{61}$, apresenta a variabilidade climática como um dos pontos-chave da fome no mundo e traz dados sobre a prevalência de desnutridos na população total (\%) no período de 2015 a 2017. Os países africanos que aparecem nas publicações elegíveis para nosso estudo (a maioria localizada na África Oriental) possuem, predominantemente, prevalências classificadas pelo estudo entre moderadamente alta (15-24,9\%), alta (25$34,9 \%$ ) e muito alta (35\% ou mais), exceto a Áfri- 
Quadro 1. Síntese dos resultados encontrados nos estudos.

\begin{tabular}{|c|c|c|c|}
\hline Categorias & $\begin{array}{c}\text { Artigos } \\
\text { relacionados }\end{array}$ & $\begin{array}{l}\text { Total de } \\
\text { artigos }\end{array}$ & Resultados encontrados \\
\hline Acesso & $\begin{array}{l}4,5,11,12,15 \\
19-28\end{array}$ & 15 & $\begin{array}{l}\text { - Falta de renda como consequência do desemprego dos agricultores que perdem } \\
\text { seus animais (pecuária), suas terras e/ou com a redução da produtividade das } \\
\text { mesmas } \\
\text { - Aumento dos preços dos alimentos básicos decorrente da queda da } \\
\text { produtividade e aumento da demanda } \\
\text { - A dificuldade na distribuição e armazenamento da produção resultando em } \\
\text { menor acesso aos alimentos }\end{array}$ \\
\hline Disponibilidade & $\begin{array}{l}1,5,11,12,15,19 \\
20-25,28,31-43\end{array}$ & 26 & $\begin{array}{l}\text { - Mudanças climáticas afetando diretamente na disponibilidade de alimentos, } \\
\text { especialmente no que diz respeito a produção de alimentos } \\
\text { - Por meio de alterações nas condições agroecológicas (aumento da temperatura, } \\
\text { aumento da frequência e severidade de eventos extremos, aumento do nível } \\
\text { dos rios, erosão dos solos, aumento das concentrações } \mathrm{d}^{\mathrm{C}} \mathrm{CO}_{2} \text { na atmosfera, } \\
\text { mudança do ciclo das águas, alterações do ciclo de vetores, etc.) que reduzem a } \\
\text { produtividade das plantações, como milho, trigo, sorgo e arroz e perda de animais } \\
\text { (peixes, gado, porcos e aves domésticas) } \\
\text { - Estudos de percepção de populações também discutiram a relação dos efeitos } \\
\text { das mudanças climáticas na produção de alimentos, assim como o crescimento da } \\
\text { população, maior competição de recursos e maior demanda de alimentos e menor } \\
\text { disponibilidade de alimentos por conta das variabilidades climáticas }\end{array}$ \\
\hline Utilização & $\begin{array}{l}12,15,20,22,25 \\
27,28,38,48\end{array}$ & 9 & $\begin{array}{l}\text { - Mudanças climáticas podem afetar o consumo de alimentos, qualidade } \\
\text { nutricional do alimento e valor social do mesmo } \\
\text {-A qualidade nutricional pode ser afetada pela redução nas concentrações de } \\
\text { diversos micronutrientes (minerais) } \\
\text { - Menor ingestão de frutas, legumes, nozes, sementes e peixes que pode ocasionar } \\
\text { deficiência de zinco, ferro, vitamina A, vitamina B12, vitamina D, proteína e } \\
\text { ômega } 3\end{array}$ \\
\hline Estabilidade & $4,5,12,25,28,50$ & 6 & $\begin{array}{l}\text { - Relação entre as mudanças climáticas afetando a redução da produção agrícola } \\
\text { e a quantidade de alimentos disponíveis no mercado o que, por consequência, } \\
\text { aumenta os preços dos alimentos no mercado, especialmente em áreas rurais que } \\
\text { tendem a ser mais sensíveis as variações climáticas }\end{array}$ \\
\hline $\begin{array}{l}\text { Estratégias de } \\
\text { adaptação e } \\
\text { mitigação }\end{array}$ & $\begin{array}{l}4,5,15,20-25 \\
28,31,34-39 \\
41,43,49,50 \\
53-55\end{array}$ & 24 & $\begin{array}{l}\text { - Implementação de políticas públicas, planos de desenvolvimento agrícola, leis de } \\
\text { proteção do mercado, abertura de mercados locais e subsídios agrícolas bem como } \\
\text { a manutenção e recuperação dos solos afetados pelas mudanças climáticas } \\
\text { - Reduzir as áreas de plantio de certas culturas; plantar variedades de culturas com } \\
\text { um período de crescimento mais curto; atrasar o início do plantio de acordo com } \\
\text { a precipitação; uso de agricultura de conservação, retirada de resíduos e uso de } \\
\text { rotações de cultura; investir em maquinaria adicional para encurtar o tempo de } \\
\text { plantio; coleta de água da chuva criando sulcos perto de áreas plantadas; aumentar } \\
\text { o uso de irrigação, aumentar os campos de cultura/plantações por meio do uso de } \\
\text { espécies não geneticamente modificadas e o aumentar a variedade de culturas e a } \\
\text { utilização de pastagens visto que estas tem a capacidade de sequestrar os carbonos } \\
\text { adicionais da atmosfera e armazenar no solo } \\
\text { - Inovações tecnológicas através da genética; biotecnologia; agronomia; uso } \\
\text { de biocombustíveis; redução na utilização de fertilizantes e contaminantes; } \\
\text { utilização de fertilizantes inorgânicos; utilização da agricultura de baixo carbono; } \\
\text { fortificação e biofortificação; sequestro de carbono orgânico; manejo de pragas; } \\
\text { rotação de culturas; intensificação de culturas; reflorestamento e irrigação } \\
\text { - União das necessidades de adaptação humana e natural relacionadas à } \\
\text { manutenção da produtividade agrícola e da integridade do ecossistema } \\
\text { (agrossilvicultura) } \\
\text { - Sistemas de alerta e alarme, e, ferramentas de monitoramento assim como a } \\
\text { elaboração de planos integrados para prevenção, proteção, resposta e reabilitação } \\
\text { das áreas expostas através da participação social } \\
\text { - Conhecimento, conscientização e comunicação como formas de adaptação às } \\
\text { mudanças climáticas através de práticas educativas e sustentáveis }\end{array}$ \\
\hline
\end{tabular}




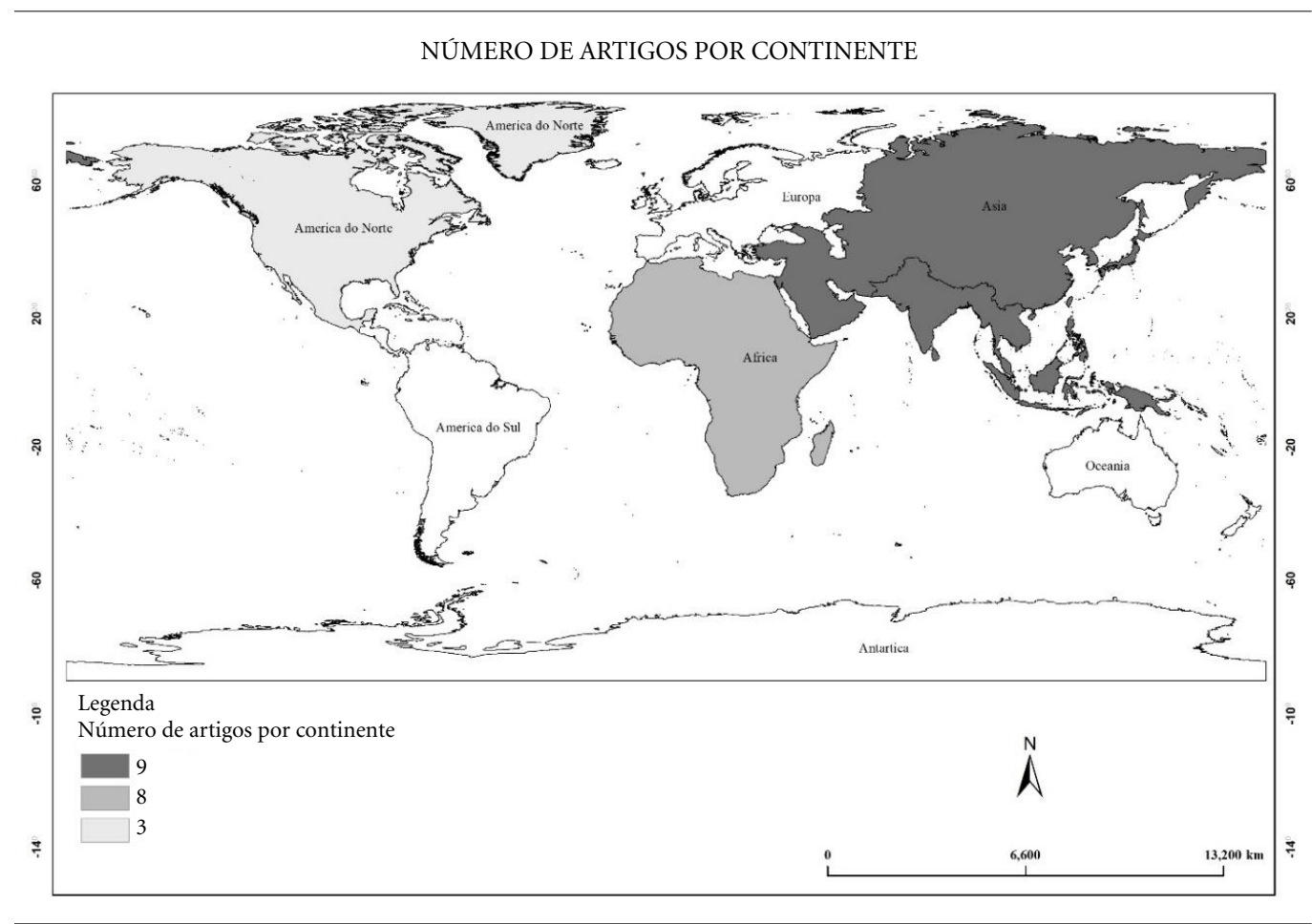

Figura 3. Distribuição das publicações elegíveis para o estudo por continente.

Fonte: Autores, 2020.

ca Ocidental e a África do Sul que apresentam prevalência moderadamente baixa $(5-14,9 \%)^{61}$. Ou seja, aproximadamente $20 \%$ da população africana passa fome por diversas causas, dentre elas os efeitos das mudanças climáticas na produção agrícola do continente.

Pode-se concluir que o continente africano possui uma gama de vulnerabilidades: social, econômica e nutricional, dentre outras não apontadas no presente estudo. Sabe-se que o risco varia de um local para outro e os impactos também podem ser desproporcionais de um local para outro. Isso se deve, principalmente, pelo fato de os locais apresentarem capacidades diferenciadas para se preparar, responder e se recuperar frente a situações adversas, além de padrões de exposição variados. Ou seja, uma alta vulnerabilidade é potencializada por uma baixa capacidade de enfrentamento/resposta. Considerando esse cenário, faz-se necessário também apontar o elevado número de desastres naturais que afetaram o continente africano nos últimos anos. Dados de 2017 apontam a África como o $3^{\text {o }}$ continente em número de desastres naturais registrados no mundo e o segundo em número de vítimas fatais por desastres naturais no ano: 1 em cada 5 vítimas fatais de desastres naturais no mundo, são do continente africano ${ }^{62}$.

\section{Ásia}

A Ásia é o maior continente do planeta e também o mais populoso, com aproximadamente $60 \%$ de toda a população mundial. A maioria dos países asiáticos estão em situação de pobreza extrema em contraste a pequenos clãs detentores de grandes riquezas, o que faz da desigualdade social um grande problema no continente.

Dentre os indicadores sociais, é importante destacar que 1 em cada 5 asiáticos é analfabeto e que a taxa de mortalidade infantil do continente chega a 115 no Afeganistão (uma das maiores do ranking mundial). Somado a isso, o continente enfrenta problemas como: mortalidade materna, violência sexual, conflitos armados, emergências de saúde pública.

Os países asiáticos que aparecem nas publicações elegíveis para nosso estudo aparecem no Mapa da Fome ${ }^{61}$, com prevalências de desnutridos na população total entre moderadamente alta $(15-24,9 \%)$ e alta $(25-34,9 \%)$, exceto a Ín- 
dia que apresentou uma prevalência classificada como moderadamente baixa (5-14,9\%). Isso nos mostra que a SAN é uma importante questão no continente $^{61}$.

Pode-se concluir que, apesar de grandes potências mundiais estarem na Ásia, o continente possui uma sobreposição de vulnerabilidades: social, econômica e nutricional, dentre outras não apontadas no presente estudo. Esta situação associada com aumento na frequência e intensidade de desastres naturais, um dos efeitos das mudanças climáticas, torna-se uma preocupação. No ano de 2017 a Ásia foi o continente com o maior número de desastres naturais registrados no mundo ( $40 \%$ do total) e com o maior número de mortes (58\%). Três dos 5 países no ranking mundial de vítimas fatais de desastres naturais são asiáticos ${ }^{62}$.

\section{SAN $x$ pobreza $x$ desigualdade social}

A relação entre as mudanças climáticas, pobreza e desigualdade é complexa, multifacetada e específica para cada contexto analisado. Os primeiros estudos surgiram em 2017 quando notou-se que as mudanças climáticas poderiam aumentar a pobreza e aprofundar as desigualdades preexistentes por meio dos seus efeitos adversos na agricultura e na saúde. Atualmente, no entanto, há consenso de que essa relação é caracterizada por um ciclo vicioso que se retroalimenta: a pobreza existente faz com que, por exemplo, as populações desfavorecidas ou mais pobres vivam em áreas mais propensas ou mais expostas a diferentes riscos, incluindo os riscos climáticos (vulnerabilidade geográfica e ambiental), da mesma maneira faz com que esses mesmos indivíduos/ grupos em desvantagem sofram desproporcionalmente com os efeitos dos riscos das mudanças climáticas ou sejam mais suscetíveis a eles, visto que possuem menos recursos para manejá-los e para se recuperar frente a estes. Uma vez afetadas, as populações mais pobres ficam ainda mais vulneráveis, exacerbando ainda mais a pobreza e desigualdade social preexistente no local/população e que as fará mais expostas ou mais propensas a novos cenários de risco ${ }^{63}$.

Somado a isso, existe também a vulnerabilidade política: grupos menos favorecidos têm menos voz e representatividade e, normalmente, são excluídos das arenas de decisão e, consequentemente, das políticas públicas ${ }^{63}$.

Os efeitos na saúde também são importantes nessa discussão pessoas vivendo em condições de pobreza são mais suscetíveis a doenças que os efeitos das mudanças climáticos ajudam a pro- pagar, incluindo as relacionadas aos vetores e à água contaminada, além de possuir menos acesso a serviços de saúde ${ }^{63}$.

Estudos mostram que as regiões mais pobres são mais severamente afetadas pelas ameaças climáticas do que as ricas. Assim como, pessoas em desvantagens social e demográfica, incluindo aqueles que enfrentam discriminação baseada no gênero, idade, raça, classe, casta, são particularmente mais afetadas pelos efeitos negativos destas. Além disso, fatores como o tipo de ocupação do indivíduo (escolha fortemente influenciada pela sua condição socioeconômica) podem intensificar os efeitos das mudanças climáticas. Exemplo: uma população que vive predominantemente da agricultura e que exerce atividades externas e totalmente dependentes do clima sofrerá mais com os efeitos das mudanças climáticas $^{63}$.

Considerando esse contexto, chama a atenção a inexistência de estudos no Brasil sobre os efeitos das mudanças climáticas na SAN, sendo que o país é considerado 1 dos 10 "hotspots" para mudanças climáticas e onde a insegurança alimentar, assim como a pobreza, é considerada um problema de saúde pública, especialmente pelas desiguais e inadequadas condições de acesso e distribuição de alimentos para a população $0^{54,64,65}$.

$\mathrm{O}$ atual sistema alimentar global impulsiona as más formas de nutrição (desnutrição, deficiências nutricionais, sobrepeso e obesidade) através de maiores emissões de gases de efeito estufa, desmatamento e degradação do solo, perda da biodiversidade, uso massivo de agrotóxicos, todos estes colaborando para as mudanças no clima e no ambiente ${ }^{47}$. E por sua vez, as mudanças climáticas causam alterações no acesso, disponibilidade, utilização e estabilidade dos sistemas alimentares afetando o estado nutricional das populações e intensificando as diversas formas de má nutrição $0^{47}$. Esta situação se torna ainda mais grave e preocupante entre os mais vulneráveis a SAN que possuem menor capacidade de adaptação, não somente às variações na produção $\mathrm{e}$ preço dos alimentos, como também aos efeitos das mudanças climáticas ${ }^{51}$.

Vale ressaltar como limitações deste estudo que a escolha de somente uma base de dados, neste caso o PubMed, pode ter resultado em perdas nesta pesquisa em termos de um número reduzido de artigos contemplados e revisados. Diante disso, se faz necessário ampliar os estudos e pesquisas sobre a relação entre mudanças climáticas, más formas de nutrição e SAN. 


\section{Conclusão}

Os resultados do estudo apontam que as mudanças climáticas afetam direta e indiretamente as quatro dimensões da SAN, especialmente em populações mais pobres ${ }^{4,50}$, e trazem para a discussão estratégias de adaptação/mitigação a esse novo cenário.

Foram considerados elegíveis para o estudo 34 artigos que contemplam a interface mudanças climáticas e SAN, no período de 2004 a 2018, o que gera uma média de 2,2 artigos por ano e chama a atenção para a lacuna existente sobre o assunto na literatura. No entanto, a relevância do tema para a agenda da SAN suscita a premência de políticas públicas de proteção social e incentivo para pesquisas, especialmente nos países mais pobres do planeta que serão os mais afetados. Essas pesquisas devem possuir uma visão holística do problema, com foco na construção de um sistema alimentar mais resiliente/resistente ao clima, e não somente para questões relacionadas à produção agrícola, visto que uma produção suficiente e sustentável de alimentos não garantirá uma população livre da fome, e das formas de má nutrição, como a desnutrição e a obesidade, apesar de resolver parte do problema ${ }^{51}$.
Chama atenção a ausência de estudos no Brasil, país que sofreu durante décadas com a fome e a desnutrição e que vivenciou progressos em relação à SAN no período de 2004 a 2013 com a implementação de políticas públicas de transferência de renda (Bolsa Família, por exemplo) e políticas públicas de promoção da SAN, que resultou na saída do país do Mapa da Fome em $2014^{66-68}$. Atualmente o Brasil vive o desmonte de políticas públicas pelo governo federal, além de fortes restrições orçamentárias para essas pastas, retrocessos que podem causar a sua volta para o Mapa da Fome ${ }^{67}$.

Conclui-se que os impactos das mudanças climáticas na SAN são reais e constituem um obstáculo ao cumprimento da agenda $2030 \mathrm{em}$ todo o planeta. Diante disso, é urgente a reestruturação do sistema alimentar, do perfil agrícola mundial e uma mudança no perfil alimentar e de consumo da população para o enfrentamento dos efeitos negativos das mudanças climáticas.

Por fim, é necessária a elaboração e implementação de políticas públicas capazes de articular diferentes agendas governamentais, independentemente da arena política no momento, visando garantir o direito humano a uma alimentação adequada e saudável.

\section{Colaboradores}

TMA Alpino e ML Mazoto trabalharam na concepção, delineamento, análise e interpretação dos dados; bem como redação, revisão e aprovação da versão a ser publicada. DC Barros e CM Freitas trabalharam na concepção bem como redação, revisão e aprovação da versão a ser publicada. 


\section{Referências}

1. Butler CD. Food security in the Asia-Pacific: climate change, phosphorus, ozone and other environmental challenges. Asia Pac J Clin Nutr 2009; 18(4):590-597.

2. Organização da Nações Unidas para a Alimentação e a Agricultura (FAO). Organização Pan-Americana da Saúde (OPAS). América Latina e Caribe. Panorama da Segurança alimentar e nutricional. Sistemas Alimentares sustentáveis para acabar com a fome e a má nutrição. Santiago: FAO; 2017.

3. Food and Agriculture Organization of the United Nations (FAO). International Fund for Agricultural Development (IFAD). The United Nations Children's Fund (UNICEF). World Food Programme (WFP). World Health Organization (WHO). The state of food security and nutrition in the world 2018. Building climate resilience for food security and nutrition. Roma: FAO; 2018.

4. Gregory PJ, Ingram JS, Brklacich M. Climate change and food security. Philos Trans R Soc Lond B Biol Sci 2005; 360(1463):2139-2148.

5. Schmidhuber J, Tubiello FN. Global food security under climate change. Proc Natl Acad Sci USA 2007; 104(50):19703-19708.

6. Brasil. Ministério do Meio Ambiente (MMA). Plano Nacional de Adaptação à Mudança do Clima. Brasília: MMA; 2016.

7. Springmann M, Daniel Mason-D'croz MA, Sherman R, Tara G, Godfray HCJ, Gollin D, Rayner M, Ballon P, Scarborough P. Global and regional health effects of future food production under climate change: a modelling study. Lancet 2016; 387(10031):1937-1946.

8. Ziska LH, Epstein PR, Schlesinger WH. Rising CO2, climate change and public change: exploring the links to plant biology. Environ Health Perspect 2009; 117:155-158.

9. Intergovernmental Panel on Climate Change (IPPC). Climate Change 2014 Synthesis Report. Washington, D.C.: IPCC; 2014.

10. Su Y-Y, Weng Y-H, Chiu Y-W. Climate change and Food Security in East Asia. Asia Pac J Clin Nutr 2009; 18(4):674-678.

11. Myers SS, Smith MR, Guth S, Golden CD, Vaitla B, Mueller ND, Dangour AD, Huybers P. Climate change and global food systems: potential impacts on food security and undernutrition. Annu Rev Public Health 2017; 38:259-277.

12. Whitmee S, Haines A, Beyrer C, Boltz F, Capon AG, Dias BFS, Ezeh A, Frumkin H, Gong P, Head P. Safeguarding human health in the Anthropocene epoch: report of the Rockefeller Foundation - Lancet Commission on Planetary Health. Lancet 2015; 386:19732028.

13. Lim SS, Vos T, Flaxman AD, Danaei G, Shibuya K, Adair-Rohani H, Amann M, Anderson HR, Andrews KG, Aryee M. A comparative risk assessment of burden of disease and injury atributable to 67 risk factors and risk fator clusters in 21 regions, 1990-2010: a systematic review analysis for the Global Burden of Disease Study 2010. Lancet 2012; 380:2224-2260.

14. Faisal IM, Parveen S. Food security in the face of climate change, population growth, and resource constraints: implications for Bangladesh. Environ Manage 2004; 34(4):487-498.
15. Lake IR, Hooper L, Abdelhamid A, Bentham G, Boxall AB, Draper A, Fairweather-Tait S, Hulme M, Hunter PR, Nichols G, Waldron KW. Climate change and food security: health impacts in developed countries. Environ Health Perspect 2012; 120(11):1520-1526.

16. Raiten DJ, Aimone AM. The intersection of climate/ environment, food, nutrition and health: crisis and opportunity. Curr Opin Biotechnol 2017; 44:52-62.

17. Jaime PC, Delmuè DCC, Campello T, Silva DO, Santos LMP. Um olhar sobre a agenda de alimentação e nutrição nos trinta anos do Sistema Único de Saúde. Cien Saude Colet 2018; 23(6):1829-1836.

18. Organização das Nações Unidas (ONU). Transformando nosso mundo: a agenda 2030 para o desenvolvimento sustentável. Brasília: ONU; 2015.

19. Lauria V, Das I, Hazra S, Cazcarro I, Arto I, Kay S, Ofori-Danson, $\mathrm{P}$, Ahmed M, Hossain MAR, Barange M, Fernandes JA. Importance of fisheries for food security across three climate change vulnerable deltas. $S c i$ Total Environ 2018; 640:1566-1577.

20. Masipa TS. The impact of climate change on food security in South Africa: Current realities and challenges ahead. Jamba 2017; 9(1):411.

21. Mugambiwa SS, Tirivangasi HM. Climate change: A threat towards achieving 'Sustainable Development Goal number two' (end hunger, achieve food security and improved nutrition and promote sustainable agriculture) in South Africa. Jamba 2017; 9(1):350.

22. Kumar M. Impact of climate change on crop yield and role of model for achieving food security. Environ $\mathrm{Mo}$ nit Assess 2016; 188(8):465.

23. Mcelhinney J. Influencing the agricultural sector to embrace adaptation to climate change, for the sake of global food security. Environ Sci Pollut Res Int 2016; 23(9):9245-9246.

24. Gross M. Food security in the times of climate change. Curr Biol 2013; 23(1):R1-R4.

25. Wheeler T, Von Braun J. Climate change impacts on global food security. Science 2013; 341(6145):508-513.

26. Douglas V, Chan HM, Wesche S, Dickson C, Kassi N, Netro L, Williams M. Reconciling traditional knowledge, food security, and climate change: experience from Old Crow, YT, Canada. Prog Community Health Partnersh 2014; 8(1):21-27.

27. Janes CR. Failed development and vulnerability to climate change in Central Asia: implications for food security and health. Asia Pac J Public Health 2010; 22(Supl. 3):236S-245S

28. Brown ME, Funk CC. Food security under climate change. Science 2008; 319(5863):580-581.

29. Fischer G, Shah M, Van Velthuizen H. Climate Change and Agricultural Vulnerability, A Special Report Prepared as a Contribution to the World Summit on Sustainable Development. Laxenburg: IIASA Rep; 2002.

30. Mugambiwa SS, Tirivangasi HM. Climate change: A threat towards achieving 'Sustainable Development Goal number two' (end hunger, achieve food security and improved nutrition and promote sustainable agriculture) in South Africa. Jamba 2017; 9(1):350.

31. Considine MJ, Siddique $\mathrm{KH}$, Foyer $\mathrm{CH}$. Nature's pulse power: legumes, food security and climate change. Exp Bot 2017; 68(8):1815-1818. 
32. Beerling DJ. Enhanced rock weathering: biological climate change mitigation with co-benefits for food security? Biol Lett 2017; 13(4):20170149.

33. Mayala BK, Fahey CA, Wei D, Zinga MM, Bwana VM, Mlacha T, Rumisha SF, Stanley G, Shayo EH, Mboera LE. Knowledge, perception and practices about malaria, climate change, livelihoods and food security among rural communities of central Tanzania. Infect Dis Poverty 2015; 4(1):21.

34. Rhodes CJ. Soil erosion, climate change and global food security: challenges and strategies. Sci Prog 2014; 97(2):97-153.

35. Godber OF, Wall R. Livestock and food security: vulnerability to population growth and climate change. Glob Chang Biol 2014; 20(10):3092-3102.

36. Ziska LH, Bunce JA, Shimono H, Gealy DR, Baker JT, Newton PC, Newton PCD, Reynolds MP, Jagadish KSV, Zhu C, Howden M, Wilson LT. Food security and climate change: on the potential to adapt global crop production by active selection to rising atmospheric carbon dioxide. Proc Biol Sci 2012; 279(1745):40974105.

37. Molyneux N, Da Cruz GR, Williams RL, Andersen R, Turner NC. Climate change and population growth in Timor Leste: implications for food security. Ambio 2012; 41(8):823-840.

38. Wesche SD, Chan HM. Adapting to the impacts of climate change on food security among Inuit in the Western Canadian Arctic. Eco Health 2010; 7(3):361-373.

39. Su YY, Weng YH, Chiu YW. Climate change and food security in East Asia. Asia Pac J Clin Nutr 2009; 18(4):674-678.

40. Gregory PJ, Johnson SN, Newton AC, Ingram JS. Integrating pests and pathogens into the climate change/ food security debate. J Exp Bot 2009; 60(10):28272838.

41. Catford J. Food security, climate change and heath promotion: opening up the streams not just helping out down stream. Health Promot Int 2008; 23(2):105108.

42. Lobell DB, Burke MB, Tebaldi C, Mastrandrea MD, Falcon WP, Naylor RL. Prioritizing climate change adaptation needs for food security in 2030. Science 2008; 319(5863):607-610.

43. Lal R. Soil carbon sequestration impacts on global climate change and food security. Science 2004; 304(5677):1623-1627.

44. Gornall J, Betts R, Burke E, Clark R, Camp J, Willett $\mathrm{K}$, Wiltshire A. Implications of climate change for agricultural productivity in the early twenty-first century. Philos Trans R Soc Lond B Biol Sci 2010; 365(1554):2973-2989.

45. McMichael AJ, Campbell-Lendrum DH, Corvalan CF, Ebi KL, Githeko AK, Scheraga JD, Woodward A, editores. Climate change and human health: risks and responses. Geneva: WHO; 2003.

46. Intergovernmental Panel on Climate Change (IPCC). Climate Change 2007: Impacts, Adaptation and Vulnerability. Contribution of Working Group II to the Fourth Assessment Report of the Intergovernmental Panel on Climate Change. Cambridge: Cambridge University Press; 2007.
47. Swinburn BA, Kraak VI, Allender S, Atkins VJ, Baker PI, Bogard JR, Ezzati M, Friel S, Goenka S, Hammond RA, Hastings G, Hawkes C, Herrero M, Hovmand PS, Howden M, Jaacks LM, Kapetanaki AB, Kasman M, Kuhnlein HV, Kumanyika S, Larijani B, Lobstein T, Long MW, Matsudo VKR, Mills SDH, Morgan G, Morshed P, Nece PM, Pan A, Patterson DW, Sacks G, Shekar M, Simmons GL, Smit W, Tootee A, Vandevijvere S, Waterlander WE, Wolfenden L, Dietz WH. The global syndemic of obesity, undernutrition, and climate change: The Lancet Commission report. Lancet 2019; 393(10173):791-846.

48. Guyot M, Dickson C, Paci C, Furgal C, Chan HM. Local observations of climate change and impacts on traditional food security in two northern Aboriginal communities. Int J Circumpolar Health 2006; 65(5):403-415.

49. Sá JCM, Lal R, Cerri CC, Lorenz K, Hungria M, Carvalho PCF. Low-carbon agriculture in South America to mitigate global climate change and advance food security. Environ Int 2017; 98:102-112.

50. Brown ME, Hintermann B, Higgins N. Markets, climate change, and food security in West Africa. Environ Sci Technol 2009; 43(21):8016-8020.

51. Organização das Nações Unidas para Alimentação e Agricultura (FAO). The stage of food security and nutrition in the world: safeguarding against economics slowdowns and downturns. Roma: FAO; 2019.

52. Hallegatte S, Bangalore M, Bonzanigo L, Fay M, Kane T, Narloch U, Rozenberg J, Vogt-Schilb A. Climate change and poverty [Internet]. Climate Change Group 2014, World Bank Group [cited 2019 mar 13]. Available from: http://www.worldbank.org/content/dam/ Worldbank/document/Climate/Climate\%20and $\% 20$ Poverty\%20Conference/D1S1_Hallegatte_CCandPov_9Fev_v6.pdf.

53. O'Mara FP. The role of grasslands in food security and climate change. Ann Bot 2012; 110(6):1263-1270.

54. Hannah L, Ikegami M, Hole DG, Seo C, Butchart SH, Peterson AT, Roehrdanz, PR. Global climate change adaptation priorities for biodiversity and food security. PLoS One 2013; 8(8):72590.

55. Palm CA, Smukler SM, Sullivan CC, Mutuo PK, Nyadzi GI, Walsh MG. Identifying potential synergies and trade-offs for meeting food security and climate change objectives in sub-Saharan Africa. Proc Natl Acad Sci 2010; 107(46):19661-19666.

56. Lipper L, Thornton P, Campbell BM, Baedeker T, Braimoh A, Bwalya M, Bwalya M, Caron P, Cattaneo A, Garrity D, Henry K, Hottle R, Jackson L, Jarvis A, Kossam F, Mann W, McCarthy N, Meybeck A, Neufeldt H, Remington T, Thi Sen P, Sessa R, Shula R, Tibu A, Torquebiau EF Climate-smart agriculture for food security. Nat Clim Chang 2014; 4(12):1068.

57. Campbell BM, Thornton P, Zougmore R, Asten P, Lipper L. Sustainable intensification: what is its role in climate smart agriculture? Curr Opin Environ Sustain 2014; 8:39-43.

58. Food and Agriculture Organization of the United Nations (FAO). Climate smart agriculture managing ecosystems for sustainable livelihoods [Internet]. [cited 2019 mar 13]. Available from: http://www.fao.org/3/a-an177e.pdf. 
59. Food and Agriculture Organization of the United Nations (FAO). Evaluation of FAO's contribution to Climate Change Adaptation and Mitigation. Final Report. Annexes [Internet]. FAO; 2015 [cited 2019 mar 13]. Available from: http://www.fao.org/3/a-bd719e.pdf.

60. Beegle K, Christiaensen L, Dabalen A, Gaddis I. Poverty in a rising Africa [Internet]. The World Bank; 2016 [cited 2019 mar 13]. Available from: https://openknowledge.worldbank.org/handle/10986/22575.

61. World Food Programme (WFP). Hunger Map 2018 [Internet]. [cited 2019 mar 13]. Available from: https://docs.wfp.org/api/documents/WFP-0000098743/ download/?_ga=2.77837533.837718209.1559262758733728121.1559262758

62. Centre for Research on the Epidemiology of Disasters (CRED). Annual Statistical Review - 2017 [Internet]. CRED; 2018 [cited 2019 mar 13]. Available from: https://www.cred.be/annual-disaster-statistical-review-2017.

63. Islam SN, Winkel J. Climate Change and Social Inequality [Internet]. DESA Working Paper; 2017 [cited 2019 mar 13]. Available from: https://www.un.org/ esa/desa/papers/2017/wp152_2017.pdf

64. Marengo JA, Jones R, Alves LM, Valverde M. Future change of temperature and precipitation extremes in South America as derived from the PRECIS regional climate modeling system. Int J Climatol 2009; 29(15):2241-2255.

65. Ambrizzi T, Rocha RP, Marengo JA, Pisnitchenko I, Alves LM. Cenários regionalizados de clima no Brasil para o Século XXI: Projeções de clima usando três modelos regionais. Relatório 3. Brasília: MMA; 2007.
66. Silva JG, Tavares L. Segurança alimentar e a alta dos preços dos alimentos: oportunidades e desafios. Segurança alimentar e nutricional. Campinas 2008; 15(1):62-75.

67. Vasconcelos FAG, Machado ML, Medeiros MAT, Neves JA, Recine E, Pasquim EM. Public policies of food and nutrition in Brazil: From Lula to Temer. Rev Nutr 2019; 32:e180161.

68. Organização das Nações Unidas para a Alimentação e a Agricultura (FAO Brasil). Estado da Segurança Alimentar e Nutricional no Brasil 2015. Agendas convergentes. Brasília: FAO Brasil; 2015.

Artigo apresentado em 13/06/2019

Aprovado em 18/11/2020

Versão final apresentada em 20/11/2020

Editores-chefes: Romeu Gomes, Antônio Augusto Moura da Silva 